\title{
Header Concept
}

National Cancer Institute

\section{Source}

National Cancer Institute. Header Concept. NCI Thesaurus. Code C43637.

A designation for a concept that indicates something about the concepts grouped under

it, but that is not intended for coding etc. (example: Neoplasm by Site). 